\title{
The Challenge of Electronic Cigarettes
}

\author{
Sutoidem Akpanudo, MD'
}

Author affiliations are

listed at the end of this

article.

Correspondence to:

Sutoidem Akpanudo, MD

Duke University

School of Medicine

sutoidem.akpanudo@duke.edu

\section{KEYWORDS}

Electronic Cigarettes, e-cigarettes, smoking cessation, smoking prevention, nicotine dependence, smoking, cigarettes

Among adults ( 18 years and older), the use of combustible tobacco products is the leading preventable cause of mortality and results in a wide range of morbidities. ${ }^{1}$ Nevertheless, social normalization and nicotine dependence associated with combustible tobacco use have made smoking cessation a very difficult undertaking. Many people who are well aware of the adverse effects of combustible tobacco use continue to smoke, even in the face of grave morbidity and death. There are many FDA approved therapies for smoking cessation, including nicotine replacement modalities and other pharmaceutical drugs, yet many people are unable to quit smoking using these therapies. It has been estimated that most quit attempts (79\%) last for less than one day. ${ }^{2}$

Since their introduction into the US market in 2006, electronic cigarettes, also known as e-cigarettes, have been seen by many to be a powerful tool against combustible tobacco use. The initial optimism that surrounded e-cigarette use in the fight against smoking has been tempered by controversies surrounding its safety, efficacy, and the involvement of tobacco companies in the production and marketing of e-cigarettes. ${ }^{3}$ This has essentially led to a situation where this very potent tool has been left to languish while the devastating health effects of combustible tobacco use rages on. Many professional healthcare societies do not have clear guidelines for their healthcare providers when it comes to e-cigarette use as a tool for smoking cessation or prevention. Consequently, many healthcare providers are uncertain about how to counsel their patient about e-cigarettes.

So how did we get here? The e-cigarette was invented by Hon Lik, a Chinese pharmacist, in 2003 to help himself quit smoking after his father died from smoking. ${ }^{4}$ Consequently, the initial purpose of e-cigarettes was to use them as a smoking cessation tool, but its potential use as a smoking prevention device has always been a consideration since its invention. As a smoking cessation tool, e-cigarettes have been shown by multiple studies to be effective for smoking cessation. ${ }^{5}$ In one large trial, e-cigarettes were more effective than nicotine patch, a first line pharmacotherapeutic agent. ${ }^{6}$ In addition, users of e-cigarettes appear to take a personal liking to these devices, increasing compliance and making them 
unique in smoking cessation therapies by being able to occupy the same psychological niche that combustible cigarettes occupy. ${ }^{7}$ Obviously, in doing so it does raise the question of replacing one evil with another, but it has been demonstrated that although e-cigarettes do produce harmful effects, including shortness of breath, exacerbation of asthma, potentially carcinogenic compounds resulting from inhalation of e-liquid chemicals, malfunction of delivery devices leading to injury to users and other unknown long-term health consequences, these are in no way near the magnitude of harm caused by the use of combustible tobacco cigarettes. ${ }^{5}$

Conversely, e-cigarettes have not been shown to be an effective smoking prevention tool. Mounting evidence suggests that e-cigarette use, especially among youths, may lead to subsequent increased risk of using combustible tobacco products. ${ }^{8}$ This may be partly because of aggressive marketing and design of certain e-cigarette products, notably by the e-cigarette manufacturer JUUL, towards the youth such that they find it more appealing and easier to use discretely owing to its flavored content and compact physical form. Notwithstanding, one has to keep in mind that by far the largest proportion of e-cigarette users are current smokers or former smokers and only a small fraction of individuals who have never smoked cigarettes ever try e-cigarettes $(5.7 \%)$ or become chronic users of e-cigarettes $(0.7 \%) .{ }^{9}$ Therefore in reality, young people who do not find smoking appealing in general, appear to be unlikely to pick up the habit of e-cigarette smoking in the first place.

Given the above premise, smoking cessation using e-cigarettes with complete cessation of combustible tobacco products should be recommended as a smoking cessation tool for current smokers, who have either failed to quit with the currently available smoking cessation therapies or are unwilling to try other therapies. This should be done with the understanding that certain long-term health consequences of e-cigarette use are currently unknown but are significantly less harmful than combustible cigarettes. This is clearly a difficult needle to thread, but one which has great potential to improve the health status of millions of individuals. This is the challenge of e-cigarettes.

\section{AUTHOR AFFILIATIONS}

1. Duke University, Durham, North Carolina

\section{REFERENCES}

1. Jha P et al. 21 st-century hazards of smoking and benefits of cessation in the United States. $\mathrm{N}$ Engl J Med. 2013;368(4):341-350.

2. Hughes JR et al.,Natural history of attempts to stop smoking. Nicotine Tob Res. 2014;16(9):11901198.

3. Stone $\mathrm{E}$, Marshall H. Tobacco and electronic nicotine delivery systems regulation. Transl Lung Cancer Res. 2019;8(1):S67-S76.

4. Demick B. A high-tech approach to getting a nicotine fix, in Los Angeles Times. 2009: Los Angeles.

5. National Academies of Sciences. Public health consequences of e-cigarettes. Eaton DL, Kwan LY, Stratton K, Editors. 2018. National Academies Press (US).

6. Hajek $\mathrm{P}$ et al. A randomized trial of e-cigarettes versus nicotine-replacement therapy. $\mathrm{N}$ Engl J Med. 2019; 380(7):629-637.

7. Steinberg $M B$ et al. E-cigarette versus nicotine inhaler: comparing the perceptions and experiences of inhaled nicotine devices. J Gen Intern Med. 2014;29(11):1444-50.

8. Leventhal AM et al. Association of electronic cigarette use with initiation of combustible tobacco product smoking in early adolescence. Jama. 2015;314(7):700-707.

9. Bao W et al. Changes in electronic cigarette use among adults in the United States, 2014-2016. Jama. 2018; 319(19):2039-2041. 